$\begin{array}{ll}\text { Research Square } & \begin{array}{l}\text { Preprints are preliminary reports that have not undergone peer review. } \\ \text { They should not be considered conclusive, used to inform clinical practice, } \\ \text { or referenced by the media as validated information. }\end{array}\end{array}$

\title{
Social inequities and visual function in pediatric ophthalmology populations: a small cross-sectional study
}

Bonnie He ( $\sim$ bonnie.he@alumni.ubc.ca)

Dalhousie University

Catherine Binda

University of British Columbia

Christine Loock

University of British Columbia

Jane Gardiner

University of British Columbia

\section{Research Article}

Keywords: Ophthalmology, Social Determinants of Health, Social Pediatrics

Posted Date: February 10th, 2022

DOI: https://doi.org/10.21203/rs.3.rs-1344245/v1

License: (c) (i) This work is licensed under a Creative Commons Attribution 4.0 International License. Read Full License 


\section{Abstract \\ Objectives}

The relationship between visual function and social determinants of health in pediatric ophthalmology patients is unclear. Our study evaluated the feasibility of screening for social determinants of health in ophthalmology clinics, assess the prevalence of these risk factors, and to clarify the relationship between social determinants of health and visual function.

\section{Methods}

An institution approved survey on social determinants of health risk factors was completed by 145 patients from five pediatric ophthalmology outpatient clinics in British Columbia. Medical charts of survey participants were reviewed to determine diagnoses and level of visual function. Descriptive statistics, univariate, and multivariate analysis were performed.

\section{Results}

Socioeconomic risk factors were present in all pediatric ophthalmology settings. $56 \%(n=81)$ of participants reported having at least one risk factor. Characteristics of poverty, including annual household income, size of support network, adverse childhood experiences, and level of parental education, were not associated with level of visual function. Food insecurity, housing instability, low income, and lack of social support were all associated with a higher adverse event scores $(\mathrm{p}<0.05)$. Patients who experienced food insecurity were more likely $(\mathrm{OR} 7.14,95 \% \mathrm{Cl} 1.47-38.44)$ to have an adverse childhood experience score of four or more compared to patients who did not have any risk factors.

\section{Conclusions}

Our study found no relationship between the level of visual function and social determinants of health risk factors. Given that all pediatric ophthalmology populations experience socioeconomic poverty, the need to establish social screening and social work support is pertinent in all pediatric ophthalmology clinics.

\section{Introduction}

Approximately 19 million of the world's children are visually impaired(1) and $6.8 \%$ of children under the age of 18 in the United States have a diagnosed eye and vision condition.(2) Good vision is crucial for a child's physical, emotional, social, and developmental well-being as the impact of having a vision disorder at any age can be felt for the remaining years of life.(3-5) At the same time, the World Health Organization (WHO) has identified social determinants of health (SDOH), which include income, food security, housing stability, academic attainment, and healthcare access, to have a far greater influence on overall health outcomes than the medical care being provided. $(6,7)$ Children are especially vulnerable to the effects of SDOH, as the number of SDOH risk factors, and the length of exposure to those risk factors, have a cumulative effect as they become older.(8)

While there have been numerous population-based studies on the prevalence and demographic risk factors of visual impairment in children, the relationship between poverty and vision remains unclear.(3,9-15) We conducted a cross-sectional study that included five different pediatric ophthalmology populations across the province of British Columbia (BC) to 1) determine whether screening for SDOH and ACEs was feasible in outpatient pediatric ophthalmology settings, 2) report the prevalence of negative socioeconomic experiences in different pediatric ophthalmology populations, and 3) investigate associations between $\mathrm{SDOH}$ and level of visual function in children with a diagnosed vision disorder. Findings from this study will help identify gaps in quality of care, guide allocation of social work resources, and demonstrate the importance of social screening that may reveal health inequities and accessibility barriers in pediatric ophthalmology.

\section{Materials And Methods}

To determine the effects of SDOH in pediatric ophthalmology patients and families, an institution-approved survey was built using current best practices reflected in the literature and included questions on demographics; social and material capital; healthcare utilization and access; food, housing, and financial security; and an optional adverse childhood experiences questionnaire.(16)

Between July 2018 and December 2019, a convenience sample of 145 patients were recruited in the waiting rooms of five different ophthalmology clinic outpatient settings: British Columbia Children's Hospital (BCCH) Ophthalmology Department, Downtown Eastside Eye Clinic (DEEC), BCCH Provincial Visually Impaired Program (VIP), Prince George (PG) Pediatric Ophthalmology Outreach Clinic, and a private practice Community Clinic (CC). Patients were recruited from multiple sites to increase the generalizability of our findings. To be eligible to for the study, participants had to be English-speaking caregivers attending an appointment with their child. At the time of recruitment, participants underwent an informed consent process prior to completing a survey about ACEs and SDOH risk factors. Ophthalmological information including visual acuity (which was measured using Snellen, Teller or Allen visual acuity charts depending on the child's ability) and eye condition were extracted from the charts of participants who completed the survey. This prospective cross-sectional study obtained ethics approval from the University of British Columbia's Clinical Research Ethics Board (H19-03822) and was conducted in accordance with the principles of the Declaration of Helsinki. 
Patients were classified into three mutually exclusive groups for visual function based on their best-corrected visual acuity and the etiology of their eye disease: normal (or functional) vision (visual acuity of 20/50 or better, which is also the provincial standard for driving);(17) impaired vision (visual acuity between $20 / 60$ and greater than $20 / 200$ ) and blind (visual acuity of $20 / 200$ or less).

Patients were also categorized into seven mutually exclusive categories for their diagnoses based on the etiology that was most responsible for their visit. These seven categories include: external (eyelid, orbit and nasolacrimal system) disease, anterior segment (cornea, conjunctiva, iris, and lens), neuroophthalmology, retina, refractive error, strabismus, and screening. The screening category referred to patients who had a normal eye exam. If a patient had multiple diagnoses, (ie. refractive error and optic disc atrophy), the diagnosis most responsible for their visit was used for classification.

All statistical analyses were performed using GraphPad Prism Version 8.4.3 (GraphPad Software, Inc., CA, US) and RStudio Version 1.2.5033 (RStudio, PBC, MA, USA). If a participant did not answer a particular question, the missing data was not included in the analysis, and the sample size was adjusted accordingly. Descriptive analyses were used to summarize categorical variables of interest in frequency and percent. Principle component analysis was used to determine which of the numerous variables captured from the survey best described the variation in the dataset. Pearson's chi-square tests were then used to investigate the relationship between clinic site, visual impairment, and demographics of the patient population including annual household income, number of people the family can turn to for support, number of ACEs, and highest level of parental formal education. P-values were adjusted using the Bonferroni method to account for multiple comparisons. Throughout analysis, $p<0.05$ was considered statistically significant. If initial Pearson's chisquared tests demonstrated significance, a post hoc multiple comparisons chi-squared test was applied to elucidate the source of significant differences. Multiple logistic regression analysis was also used to examine the relationship of a priori specified SDOH variable of interest and having an ACEs score of four or more. Crude and adjusted odds ratios, as well as $95 \%$ confidence intervals (Cls) were reported.

\section{Results}

Our survey's respondents came from diverse regions of the province and reflected a heterogeneous distribution of ocular conditions. Of the 145 participants, 45 patients were from $\mathrm{BCCH}$, a tertiary care ophthalmology clinic, while 32 patients were from VIP, a clinic for children with low vision. Twentysix patients came from DEEC, a pediatric ophthalmology clinic operating in one of Canada's poorest neighbourhoods, while 21 patients were from PG, a rural outreach clinic that serviced patients from northern regions of BC. Finally, 21 patients from $\mathrm{CC}$, which was a general pediatric ophthalmology clinic in the community. Most of the patients had a family size of four to six members (62.1\%) and about a third (37.2\%) of the patients identified as being in a visible, ethnic or cultural minority. Over a quarter (25.5\%) of patients had parents whose highest level of formal education was a high school diploma or less. $28.3 \%$ of respondents had an annual household income below $\$ 40,000$, which is the poverty income line for a family of four in BC.(18) This figure is higher than the BC and Canadian child poverty rate of $20.3 \%$ and $19.6 \%$ respectively.(19) The majority of patient families ( $94.4 \%$ ) had a primary healthcare provider, which is in contrast to the provincial and national average of $15.3 \%$ and $18.2 \%$ respectively for families who did not have a family doctor.(20) The demographic characteristics of the study participants are listed in the supplementary eTable 1.

\section{Differences between clinics}

Apart from the Visually Impaired Program, in which patients were referred to by a pediatric ophthalmologist after confirmation of having a diagnosed visual impairment, the majority of patients seen at the other four clinics had normal vision (Supplementary eFigure 1). The DEEC and CC had patient populations of similar visual function, with refractive errors being the most common diagnosis at these two clinics and neither having patients who were blind. Likewise, BCCH and PG also had similar patient population visual function profiles, with both having a wider variety of vision problems (Supplementary eFigure 2).

Although the DEEC and CC had patients of similar visual function and diagnoses, compared to patients from the CC, patients at the DEEC were more likely to experience low household income $(p=<0.0001)$, few social supports $(p<0.01)$, high ACEs $(p=<0.0001)$, and low levels of parental education ( $p<0.001)$ (Figure 1). In contrast, while the $\mathrm{BCCH}$ and PG pairing also had near-identical patient population profiles in terms of visual function and diagnoses, there were no differences in negative social determinants experienced by patients between these two clinics (Figure 1).

\section{Differences between visual function}

Excluding the VIP clinic in which all patients were blind or had impaired vision, $1.8 \%(n=2)$ of the study population were blind and $3.5 \%$ ( $n=4)$ had impaired vision. The top three causes of blindness for the entire study population were neuroophthalmological $(63.6 \%, n=14)$, retinal $(31.8 \%, n=7)$ and anterior segment $(4.5 \%, n=1)$ diseases (Supplementary eFigure 3$)$. This is consistent with literature reports that have listed cortical visual impairment, optic nerve hypoplasia, retinopathy of prematurity, optic atrophy, albinism and coloboma as being the top causes of childhood blindness.(21-23) Common causes of impaired vision included retinal $(50 \%, n=8)$ and neuroophthalmological $(37.6 \%, n=6)$ diseases. Interestingly, there were no associations between the level of visual function and SDOH risk factors, including annual household income, social support, ACEs or level of parental education. (Figure 2).

\section{Relationship between ACEs and SDOH}

Of the 145 participants, 103 (71.0\%) answered the ACEs questionnaire. Four (food security, housing stability, income, and social support) of the five SDOH risk factors had a statistically significant $(p<0.05)$ negative association with ACEs (Table 1). There was no significant relationship between the level of parental education and ACEs. After adjusting for SDOH co-variates, only one of the five SDOH risk factors (food insecurity) was associated with a 
statistically significant greater likelihood (OR 5.5, 95\% Cl 1.24-25.25) of an ACEs scores of four or more compared to patients who did not have any SDOH factors (Table 2).

\section{Discussion}

Visual impairment is one of the most prevalent causes of childhood disability,(2) and while social determinants of health are known barriers to ophthalmic follow-up care,(24-26) there have been mixed reports of their influence on visual function in the pediatric ophthalmology population. $(3,9,12-15)$

Our study demonstrates that social screening for SDOH and ACEs can be feasible in an outpatient setting, and that appropriate resources can be made available in the clinic to address concerns arising from patients with significant negative SDOH and/or high ACEs scores. The results of this study found socioeconomic risk factors, such as low income, social isolation, lack of a fixed address, and limited access to nutritious foods, to be present in all pediatric ophthalmology settings, and ophthalmologists should be cognizant of this when considering barriers to care and treatment compliance. Statistically significant relationships between childhood socioeconomic vulnerability and ACEs were also detected on univariate and multivariate analyses.

We did not find any associations between visual function and SDOH risk factors, a finding that has also been reported in the literature.(9) Patients with visual impairment were no more likely to come from households of lower education, of lower income, or of lower social network than those with no visual impairment. Moreover, when we compared SDOH between clinics that had patients with similar visual function, no relationships between the level of visual impairment or diagnoses and the various SDOH risk factors were observed. For example, the DEEC and CC had near-identical types of patients in which patients from these two clinics were usually referred by family doctors, pediatricians or optometrists and often did not require tertiary care, yet there were significant differences in social determinants experienced between the two clinic patient populations.

The strengths of our study include the successful implementation of our screening questionnaire for SDOH risk factors in different pediatric ophthalmology outpatient clinic settings as well as the heterogeneity of pediatric eye conditions captured. Some limitations in our results include the relatively small sample size, and the possibility of recall and reporting bias in self-reported survey responses. In addition, while this study examined numerous social and financial factors related to health, not all possible SDOH risk factors were included. Finally, because participants represented in our convenience sample were those who were present for their appointments, patients who face additional accessibility barriers to attending appointments may not have been represented in our study, and thus generalizing our results should be done with caution.

Overall, poverty can significantly alter a child's health and life trajectory, and public and professional stakeholders need to recognize the impacts of material deprivation, social isolation, and adverse childhood experiences on pediatric ophthalmology patients and their families. While SDOH risk factors were found in all our pediatric ophthalmology clinic settings, they were not associated with level of visual function or diagnosis. Future directions include larger studies to confirm the findings observed and evaluating the utility of social work support in pediatric ophthalmology outpatient clinics.

\section{Declarations}

\section{Acknowledgments and Disclosures:}

This work was conducted on the traditional, ancestral, and unceded lands of the Coast Salish and Carrier peoples. Funding was provided by the Office of Pediatric Surgical Evaluation and Innovation (UBC).

None of the authors have any conflicts of interest or financial disclosures. This project was approved by the University of British Columbia's Clinical Research Ethics Board (H19-03822).

\section{References}

1. Sheet F. Visual impairment and blindness. 2013-11-06].http://www.who.int/me-diacentre/factsheets/fs282/en/index.html [Internet]

2. Vision Health Initiative Fast Facts [Internet]. cited June 16 2020]. Available from: https://www.cdc.gov/visionhealth/basics/ced/fastfacts.htm

3. Cumberland PM, Rahi JS, for the UK Biobank Eye and Vision Consortium. Visual function, social position, and health and life chances: The UK biobank study. JAMA Ophthalmol [Internet]. 2016 [cited 1/11/2020];134(9):959-66. Available from: https://doi.org/10.1001/jamaophthalmol.2016.1778

4. Repka MX. A close look at pediatric eye disease. Ophthalmology [Internet]. 2014;121(3):617-8. Available from: https://doi.org/10.1016/j.ophtha.2013.12.038

5. Pezzullo L, Streatfeild J, Simkiss P, Shickle D. The economic impact of sight loss and blindness in the UK adult population. BMC Health Services Research [Internet]. 2018;18(1):63. Available from: https://doi.org/10.1186/s12913-018-2836-0

6. Social Determinants of Health [Internet]. cited June 12 2020]. Available from: http://www.who.int/social_determinants/en/

7. Heiman HJ, Artiga S. Beyond health care: The role of social determinants in promoting health and health equity. Health [Internet]. 2015;20(10):1-10.

8. Power C, Manor O, Matthews S. The duration and timing of exposure: Effects of socioeconomic environment on adult health. Am J Public Health [Internet]. 1999 Jul;89(7):1059-65.

9. Pai AS, Wang JJ, Samarawickrama C, Burlutsky G, Rose KA, Varma R, Wong TY, Mitchell P. Prevalence and Risk Factors for Visual Impairment in Preschool Children: The Sydney Paediatric Eye Disease Study. [Internet].; 2011. 1495 p. Available from: 
http://www.sciencedirect.com.ezproxy.library.ubc.ca/science/article/pii/S0161642011000601 DOI: https://doi-

org.ezproxy.library.ubc.ca/10.1016/j.ophtha.2011.01.027

10. Friedman DS, Repka MX, Katz J, Giordano L, Ibironke J, Hawes P, Burkom D, Tielsch JM. Prevalence of Decreased Visual Acuity among Preschool-Aged Children in an American Urban Population: The Baltimore Pediatric Eye Disease Study, Methods, and Results. [Internet].; 2008. 1786 p. Available from:

http://www.sciencedirect.com.ezproxy.library.ubc.ca/science/article/pii/S0161642008003151 DOI: https://doi-

org.ezproxy.library.ubc.ca/10.1016/j.ophtha.2008.04.006

11. Prevalence and Causes of Visual Impairment in African-American and Hispanic Preschool Children: The Multi-Ethnic Pediatric Eye Disease Study. [Internet].; 2009. 1990 p. Available from: http://www.sciencedirect.com.ezproxy.library.ubc.ca/science/article/pii/S0161642009002917 DOI: https://doi-org.ezproxy.library.ubc.ca/10.1016/j.ophtha.2009.03.027

12. Lu Q, Zheng Y, Sun B, Cui T, Congdon N, Hu A, Chen J, Shi J. A Population-based Study of Visual Impairment Among Pre-school Children in Beijing: The Beijing Study of Visual Impairment in Children. [Internet].; 2009. 1075 p. Available from:

http://www.sciencedirect.com/science/article/pii/S0002939408009021 DOI: https://doi.org/10.1016/j.ajo.2008.11.021

13. Ekpenyong, B., Naidoo, K., Ahaiwe, K., Ezenwankwo, O., Ndukwe, O., Ogar, E., Ekanem,E. Visual status and prevalence of eye disorders among schoolage children in southern nigeria. African Vision and Eye Health [Internet]. 2017;76(1) Available from:

https://avehjournal.org/index.php/aveh/article/view/377

14. Robaei D, Huynh SC, Kifley A, Mitchell P. Correctable and Non-Correctable Visual Impairment in a Population-Based Sample of 12-Year-Old Australian Children. [Internet].; 2006. 112 p. Available from: http://www.sciencedirect.com.ezproxy.library.ubc.ca/science/article/pii/S0002939406003370 DOI: https://doi-org.ezproxy.library.ubc.ca/10.1016/j.ajo.2006.02.042

15. Bountziouka V, Cumberland PM, Rahi JS. Trends in visual health inequalities in childhood through associations of visual function with sex and social position across 3 UK birth cohorts. JAMA Ophthalmol [Internet]. 2017 [cited 1/11/2020];135(9):954-61. Available from:

https://doi.org/10.1001/jamaophthalmol.2017.2812

16. Felitti VJ, M.D., FACP, Anda RF, M.D., MS, Nordenberg D, M.D., Williamson DF, M.S., PhD, Spitz AM, M.S., MPH, Edwards V, B.A., Koss MP, PhD., Marks JS, M.D., MPH. Relationship of childhood abuse and household dysfunction to many of the leading causes of death in adults: The adverse childhood experiences (ACE) study. Am J Prev Med [Internet]. 1998;14(4):245-58. Available from: https://doi.org/10.1016/S0749-3797(98)00017-8

17. 22 - Vision Impairments [Internet]. cited June 16 2020]. Available from: https://www2.gov.bc.ca/gov/content/transportation/driving-and-cycling/drivermedical/driver-medical-fitness/driver-medical-fitness-information-for-medical-professionals/ccmta-medical-standards-bc-specific-guidelines-quickaccess/22-vision-impairments\#22.6.1

18. James C. BC Budget 2019 [Internet]. February 19 cited August 4 2021]. Available from:

https://www.livingwageforfamilies.ca/bc_budget_2019\#: :text=As\%20the\%20BC\%20Poverty\%20Reduction,for\%20the\%20full\%20benefit\%20amounts.

19. 2018 BC Child Poverty Report Card [Internet]. cited June 16 2020]. Available from: https://www.sparc.bc.ca/wp-content/uploads/2018/12/1.15-FirstCall-BC-Child-Poverty-Report-Card-2018.pdf

20. Primary health care providers, 2017 [Internet]. cited June 21 2020]. Available from: https://www150.statcan.gc.ca/n1/pub/82-625x/2019001/article/00001-eng.htm

21. Al-Wadani F, Khandekar R, Al-Hussain MA, Alkhawaja AA, Khan MS, Alsulaiman RA. Magnitude and causes of low vision disability (moderate and severe visual impairment) among students of al-noor institute for the blind in al-hassa, saudi arabia: A case series. Sultan Qaboos Univ Med $\mathrm{J}$ [Internet]. 2012 Feb;12(1):62-8.

22. Knappe S, Schittkowski M, Schröder W, Hopkins A, Fichter N, Guthoff R. The currently most common causes of childhood blindness in kinshasa (d. R. congo). Klin Monbl Augenheilkd [Internet]. 2007 Jul;224(7):597-602.

23. Kong L, Fry M, Al-Samarraie M, Gilbert C, Steinkuller PG. An update on progress and the changing epidemiology of causes of childhood blindness worldwide. J AAPOS [Internet]. 2012 Dec;16(6):501-7.

24. Su Z, Marvin EK, Wang BQ, van Zyl T, Elia MD, Garza EN, Salchow DJ, Forster SH. Identifying barriers to follow-up eye care for children after failed vision screening in a primary care setting. [Internet].; 2013. 385 p. Available from:

http://www.sciencedirect.com/science/article/pii/S1091853113002176 DOI: https://doi.org/10.1016/j.jaapos.2013.05.008

25. Williams S, Wajda BN, Alvi R, McCauley C, Martinez-Helfman S, Levin AV. The challenges to ophthalmologic follow-up care in at-risk pediatric populations. [Internet].; 2013. 140 p. Available from: http://www.sciencedirect.com/science/article/pii/S1091853113000785 DOI:

https://doi.org/10.1016/j.jaapos.2012.11.021

26. Congdon NG, Ruiz S, Suzuki M, Herrera V. Determinants of pediatric cataract program outcomes and follow-up in a large series in mexico. Journal of Cataract \& Refractive Surgery [Internet]. 2007;33(10) Available from:

https://journals.Iww.com/jcrs/Fulltext/2007/10000/Determinants_of_pediatric_cataract_program.42.aspx

\section{Tables}

Table 1: Correlates of SDOH ${ }^{\dagger}$ factors and $\mathrm{ACEs}^{\ddagger}$.

The correlation between different SDOH factors and ACEs score were determined. Negative SDOH risk factors were associated with higher ACEs scores. 
${ }^{\dagger}$ Social Determinants of Health

\begin{tabular}{lllll}
\hline SDOH FACTORS vs. ACEs & Correlation $\rho$ & $\mathbf{9 5 \%}$ CI $^{\S}$ & P Value & Sample Size \\
\hline Food security & -0.344 & $-0.508--0.155$ & 0.0004 & 103 \\
\hline Housing & -0.261 & $-0.437--0.065$ & 0.0078 & 103 \\
\hline Income & -0.387 & $-0.545--0.203$ & 0.0001 & 102 \\
\hline Social support & -0.287 & $-0.461--0.092$ & 0.0035 & 102 \\
\hline Parental Education & -0.140 & $-0.331--0.062$ & 0.1604 & 102
\end{tabular}

${ }^{\ddagger}$ Adverse Childhood Experiences

${ }^{\S}$ Confidence Interval

Table 2: Crude and adjusted odds ratios for patients with a $\mathrm{SDOH}^{\dagger}$ risk factor and $\mathrm{ACEs}^{\ddagger} \geq 4$.

Multivariate analyses were conducted to determine the likelihood for patients with a SDOH risk factor to have an ACEs score $\geq 4$ compared to patients with no SDOH risk factors.

\begin{tabular}{|c|c|c|c|c|c|c|}
\hline & $\mathrm{ACE} \geq 4$ & $\mathrm{ACE}<4$ & $\begin{array}{l}\text { Crude } \\
\text { Odds Ratio }\end{array}$ & $\begin{array}{l}\text { Crude } \\
95 \% \mathrm{CI}^{\S}\end{array}$ & $\begin{array}{l}\text { Adjusted } \\
\text { Odds ratio }\end{array}$ & $\begin{array}{l}\text { Adjusted } \\
95 \% \mathrm{CI}\end{array}$ \\
\hline Jumber of Subjects & 13 & 90 & --- & --- & --- & --- \\
\hline Jo SDOH risk factors (\%) & 7.69 & 25.56 & 1.00 & Reference & 1.00 & Reference \\
\hline "ood insecurity ${ }^{\top}(\%)$ & 53.85 & 12.22 & 8.38 & $2.39-30.82$ & $5.50^{*}$ & $1.24-25.25$ \\
\hline Iousing instability (\%) & 15.38 & 1.11 & 16.18 & $1.44-365.30$ & 3.71 & $0.26-97.76$ \\
\hline Innual income $\mathbb{\pi}^{\pi} \leq 40 \mathrm{k}(\%)$ & 61.54 & 23.33 & 5.18 & 1.56- 18.79 & 2.97 & $0.67-12.99$ \\
\hline jocial support" $\leq 4$ people $(\%)$ & 53.85 & 23.33 & 3.78 & $1.14-12.96$ & 1.30 & 0.24-5.95 \\
\hline 'arental education secondary & 30.77 & 47.78 & 0.49 & $0.12-1.61$ & 0.50 & $0.10-2.12$ \\
\hline
\end{tabular}

${ }^{\dagger}$ Social Determinants of Health

${ }^{\ddagger}$ Adverse Childhood Experiences

${ }^{\S}$ Confidence Interval

" Variables odds ratios were adjusted for

* significant $(\mathrm{p}<0.05)$

Supplementary eTable 1: Characteristics of study participants.

The demographic characteristics of the 145 pediatric ophthalmology patients who participated in the study. (BCCH: British Columbia Children's Hospital; VIP: Visual Impaired Program; DEEC: Downtown Eastside Eye Clinic; PG: Prince George; CC: Community Clinic.)

Characteristics

Ophthalmology Clinic

$\mathrm{BCCH}$
$\mathrm{VIP}$

DEEC

PG

$\mathrm{CC}$

Family size

1-3 members

4-6 members

$>6$ members

Did not answer

Minority

Yes

No

Did not answer

Highest level of education

Some high school

Secondary/high school diploma

CEGEP or other non-university certificate or diploma Apprenticeship or other trades certificate or diploma University certificate or diploma below bachelor level
Participant numbers (n, \%)
45, (31.0)

$32,(22.1)$

26, (17.9)

21, (14.5)

$21,(14.5)$

45, (31.5)

90, (62.1)

8, (5.5)

2, (1.4)

$54,(37.2)$

77, (53.1)

$14,(9.7)$

22, (15.2)

15, (10.3)

29, (20.0)

$9,(6.2)$

19 , (13.1) 
University certificate or diploma: Bachelor's

35, (24.1)

Advanced degree (i.e. Masters, JD, MD, PhD)

13, (9.0)

Did not answer

3, (2.1)

Annual Income

$\$ 0-20,000$
$\$ 20,000-40,000$
$\$ 40,000-60,000$
$\$ 60,000-80,000$
$\$ 80,000-100,000$
$\$ 100,000-120,000$
$\$ 120,000+$
Did not answer

26, (17.9)

$15,(10.3)$

16, (11.0)

$16,(11.0)$

26, (17.9)

$15,(10.3)$

25, (17.2)

$6,(4.1)$

Family doctor / primary healthcare provider

Yes

137, (94.5)

No

7, (4.8)

Did not answer

$1,(0.7)$

\section{Figures}

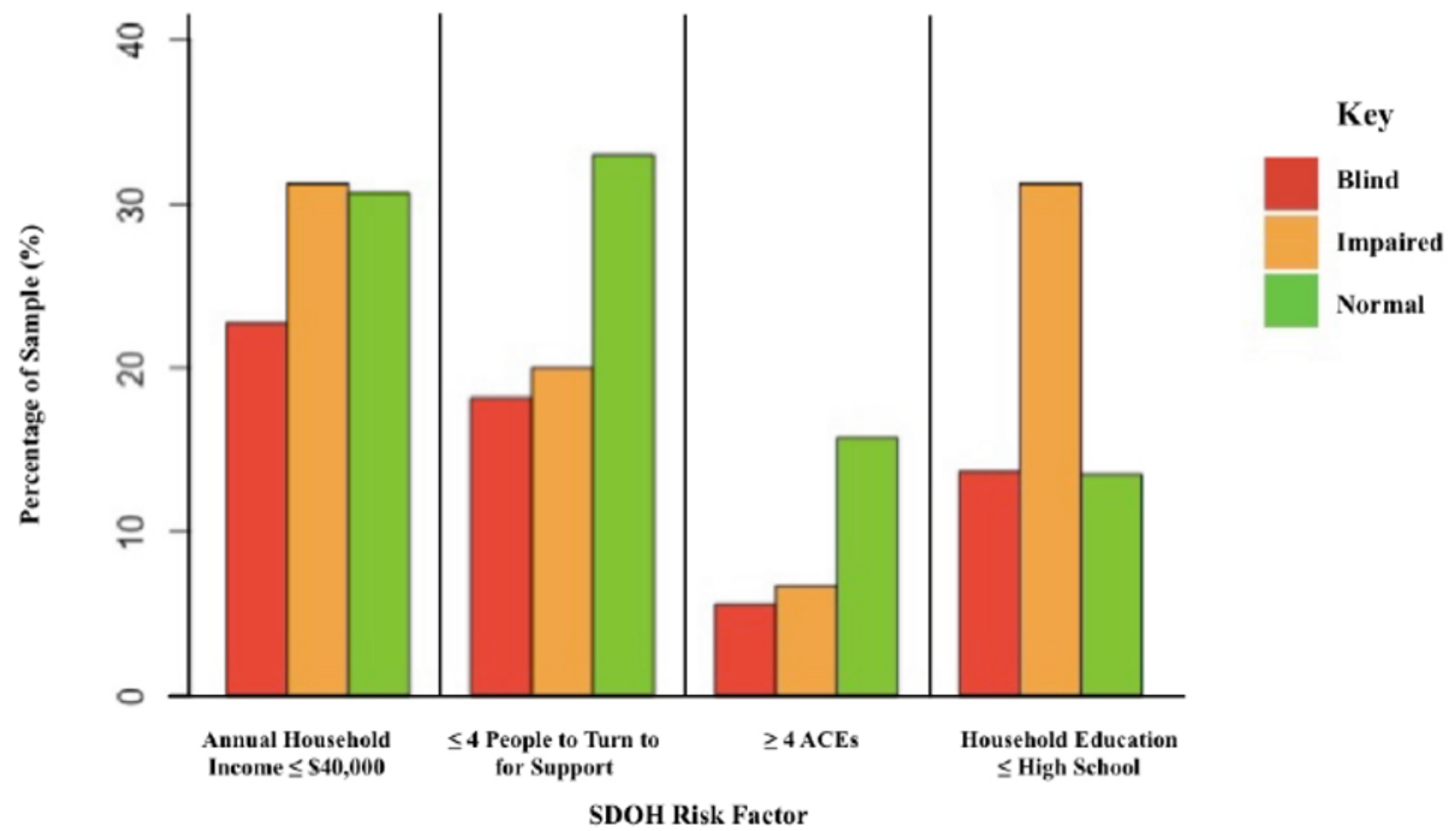

Figure 1

$\mathrm{SDOH}^{\dagger}$ Risk Factor by Visual Function.

The percentage of the participants experiencing SDOH risk factors (low annual household income, low social support, high ACEs, and low levels of formal education) is reported for each visual function group. In each cluster of columns, red is blind (visual acuity 20/200 or less) vision, orange is impaired vision (visual acuity between 20/60 and greater than 20/200), and green those with normal vision (visual acuity of 20/50 or better).

${ }^{\dagger}$ Social Determinants of Health

‡ Adverse Childhood Experiences 


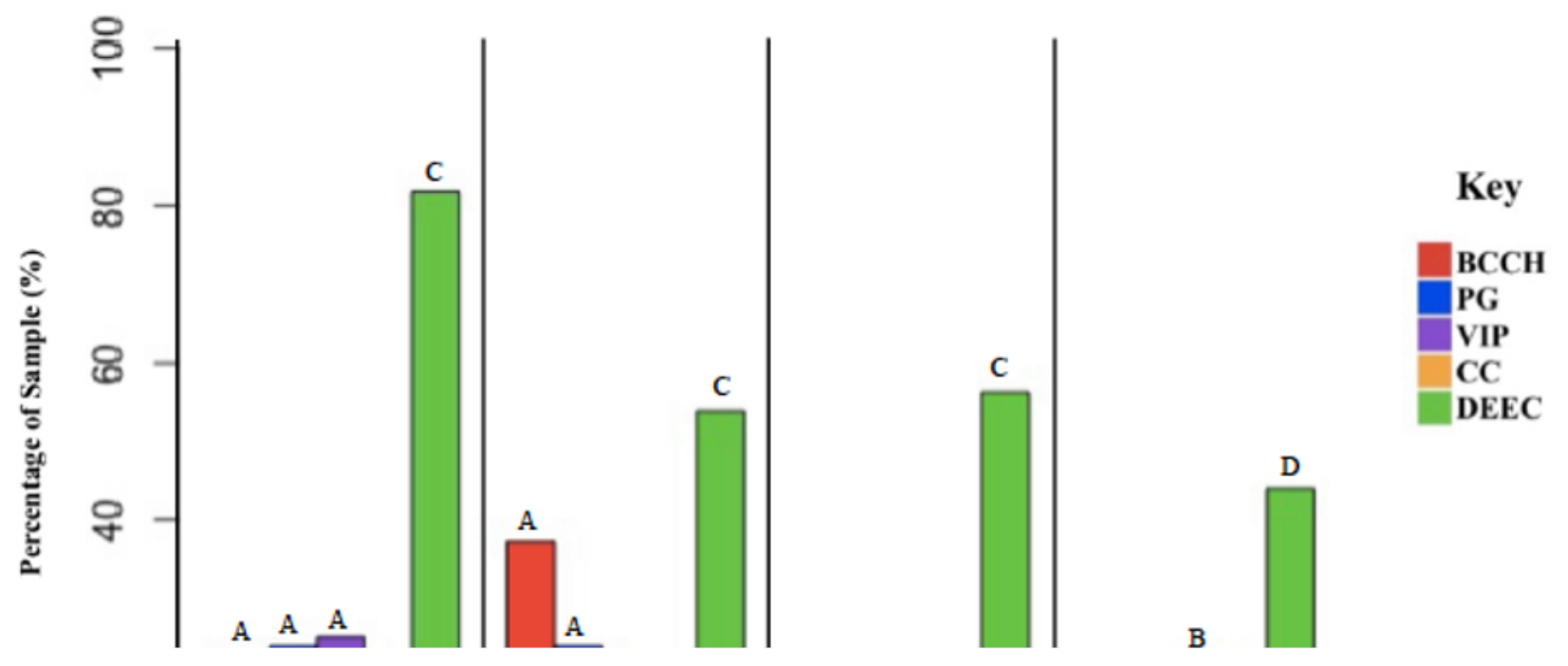

Figure 2

\section{$\mathrm{SDOH}^{\dagger}$ Risk Factor by Clinic Site.}

The percentage of the participants experiencing SDOH risk factors (low annual household income, low social support, high ACEs ${ }^{\ddagger}$, and low levels of formal education) is reported for each clinic site. In each cluster of columns, red represents British Columbia Children's Hospital (BCCH) patients, blue Prince George (PG) patients, purple Visual Impaired Program (VIP) patients, yellow Community Clinic (CC) patients, and green Downtown East Side Eye Clinic (DEEC) patients. For each cluster of columns, letters above the columns indicate significant differences in the distribution of the SDOH risk factor between clinics, as determined using a Pearson's chi-squared test. Columns that share a given letter are not significantly different from each other, while columns that do not share a common letter differ significantly. (For example, there's no significance difference between BCCH and PG for household education as they both share letter "A," and there's also no significant difference between BCCH and CC for household education because they both share letter "C.")

${ }^{\dagger}$ Social Determinants of Health

${ }^{\ddagger}$ Adverse Childhood Experiences

\section{Supplementary Files}

This is a list of supplementary files associated with this preprint. Click to download.

- Feb11Version4BCCHSurgeryandSocietyupdatedsurvery.docx

- SupplementaryeFigure1.png

- SupplementaryeFigure2.png

- SupplementaryeFigure3.jpg 